\title{
Correspondence analysis of raw data
}

\author{
Michael Greenacre \\ Department of Economics and Business \\ Universitat Pompeu Fabra
}

Ramon Trias Fargas, 25-27

08005 Barcelona

SPAIN

E-mail: michael@upf.es

\begin{abstract}
Correspondence analysis has found extensive use in the social and environmental sciences as a method for visualizing the patterns of association in a table of frequencies. Inherent to the method is the expression of the frequencies in each row or each column relative to their respective totals, and it is these sets of relative frequencies (called profiles) that are visualized. This "relativization" of the frequencies makes perfect sense in social science applications where sample sizes vary across different demographic groups, and so the frequencies need to be expressed relative to these different bases in order to make these groups comparable. But in ecological applications sampling is usually performed on equal areas or equal volumes so that the absolute abundances of the different species are of relevance, in which case relativization is optional. In this paper we define the correspondence analysis of raw abundance data and discuss its properties, comparing these with the regular correspondence analysis based on relative abundances.
\end{abstract}

Keywords: Abundance data, biplot, profiles, visualization. 


\section{Introduction}

Correspondence analysis (CA) and its variants - multiple, joint, subset and canonical correspondence analysis - have found acceptance and application by a wide variety of researchers in different disciplines, notably the social and environmental sciences (for an up-todate account, see Greenacre, 2007). The method has also appeared in the major statistical software packages, for example SPSS, Minitab, Stata, SAS, Statistica and XLSTAT, and it is freely available in several implementations in R (R Development Core Team, 2007) - for example, the ca package by Nenadić and Greenacre (2007) and the vegan package by Oksanen et al. (2006). The method is routinely applied to a table of non-negative data to obtain a spatial map of the important dimensions in the data, where proximities between points and other geometric features of the map indicate associations between rows, between columns and between rows and columns.

In the social science context where the method originated, CA is typically applied to crosstabulations between two or more categorical variables, for example a demographic variable such as education level cross-tabulated against responses to a question in an opinion survey. Because the sample sizes in the demographic groups are different, a valid comparison between these groups is achieved by expressing the response frequencies relative to their respective sample sizes, a process which we call relativization. These vectors of relative frequencies are called profiles in CA, and it is the profiles that are visualized in the resulting maps.

This technology has been transferred 'as is' to ecological applications, but it is frequently the case that ecological sampling is conducted on physically equal-sized samples, either fixed areas or fixed volumes. The Bray-Curtis index, for example, which is used to measure similarity between samples in terms of their species abundances, does not relativize the data, but aggregates absolute differences between raw abundances. Bray-Curtis measures of similarity or 
dissimilarity could be computed on relative abundance data, however, if it were deemed important to do so - the point is that the relativization step is optional in this ecological context, and not compulsory. CA inherently analyzes profiles, so the question arises how CA functions when the raw abundance levels are of interest (i.e., the size of the data) as well as the relative abundances (i.e., the shape). In this report we define the CA of raw 'unrelativized' abundance data and compare its properties to those of regular CA, with an illustration on a benthos data set from the North Sea.

\section{Correspondence analysis of relative and raw abundances}

Consider the data in Table 1 (only part of this 32-column wide table is shown) - these are typical ecological abundance data, with some species occurring very rarely and others at most or all of the sites. For purposes of visualization this table is considered as a set of rows and the question is whether we want to depict the values in each row in their raw form or relative to their respective total. Regular CA would visualize them in their relative form; that is, the profiles of each row are calculated, chi-square distances are computed between the profiles, and the profiles are then weighted by their margins in the dimension-reduction step to achieve the lowdimensional map of the sites. Species points can then be displayed as unit points and the resulting map is a biplot, called the (row) asymmetric map by Greenacre (2007) - see Figure 1. The algebraic definition of the analysis is as follows, assuming the raw data matrix is denoted by $\mathbf{N}$. The row profiles visualized in Figure 1 are the rows of Table 1 divided by their respective row totals. CA is invariant to the grand total of the table, and the notation is simplified if we regard the initial data matrix as the matrix $\mathbf{P}=\left[p_{i j}\right]$ where $p_{i j}=n_{i j} / n$, where $n$ is the grand total of the table. Let the row and column marginal totals of $\mathbf{P}$ be the vectors $\mathbf{r}$ and $\mathbf{c}$ respectively - these are the weights, or masses, associated with the rows and columns. Let $\mathbf{D}_{r}$ and $\mathbf{D}_{c}$ be the diagonal matrices of these masses. The row profiles are then rows of the matrix $\mathbf{D}_{r}^{-1} \mathbf{P}$ and the 
computational algorithm to obtain the solution, using the singular value decomposition (SVD), is as follows:

1. Center the row profiles with respect to their average $\mathbf{c}^{\top}$, then premultiply by $\mathbf{D}_{r}^{1 / 2}$ to weight the profiles by their masses, and postmultiply by $\mathbf{D}_{c}^{-1 / 2}$ to engender the chi-square metric between rows:

$$
\mathbf{S}=\mathbf{D}_{r}^{1 / 2}\left(\mathbf{D}_{r}^{-1} \mathbf{P}-\mathbf{1} \mathbf{c}^{\top}\right) \mathbf{D}_{c}^{-1 / 2}
$$

2. Calculate the SVD: $\mathbf{S}=\mathbf{U D}_{\sigma} \mathbf{V}^{\top}$ where $\mathbf{U}^{\top} \mathbf{U}=\mathbf{V}^{\top} \mathbf{V}=\mathbf{I}$.

3. Principal coordinates of rows: $\quad \mathbf{F}=\mathbf{D}_{r}^{-1 / 2} \mathbf{U D}_{\sigma}$.

4. Standard coordinates of columns: $\mathbf{Y}=\mathbf{D}_{c}^{-1 / 2} \mathbf{V}$.

(For the distinction between principal and standard coordinates, see Greenacre (2007).) The analysis of the raw abundances follows much the same scheme as that of the relative abundances described above. But no division by row totals is performed and the chi-square distance is determined by the averages of the raw abundances across rows. There are no differential weights of the rows, because using the raw abundances already includes the absolute level of each site in the description vector. To distinguish the two alternatives here, we call this analysis $C A$-raw as opposed to the regular CA, CA-relative, defined above by (1)-(4). To put CA-raw on a comparable scale to CA-relative, each row of $\mathbf{P}$ is divided by (1/I), where (1/I) is the constant row mass (as opposed to CA-relative, where each row of $\mathbf{P}$ is divided by the variable row mass); that is, the equivalent of the matrix of row profiles $\mathbf{D}_{r}^{-1} \mathbf{P}$ is $I \mathbf{P}$. This is equivalent to taking the original rows of the matrix and dividing them all by the constant $n / I$, the total abundance averaged over the $I$ rows. Some of these transformed rows will sum to more than 1 and others to less, but the (unweighted) average row is still $\mathbf{c}^{\top}$, since $(1 / I) \mathbf{1}^{\top}(I \mathbf{P})=\mathbf{c}^{\top}$. Hence, in this scale, the centering is the same as CA-relative and the chi-square metric is still based on the inverse of the matrix $\mathbf{D}_{c}$ - this shows that the essential difference between CA-raw and CA- 
relative is in the definition and weighting of the points, not in the form of the distance function between them. Thus the only change necessary in the algorithmic scheme (1)-(4) to perform CA-raw is to replace $\mathbf{D}_{r}$ throughout by $(1 / I) \mathbf{I}$.

\section{Application and results}

As is well known, in CA-relative an $I \times J$ table has dimensionality $K=\min \{I-1, J-1\}$, which in this example is 22 . The inertia, defined as the sum of squares of the matrix $\mathbf{S}$ in (1), measures the total variance in the table, and is equal to the sum of the squared singular values $\sigma_{k}^{2}, k=1, \ldots, K-$ in this example the total inertia is 3.176. The two-dimensional CA solution shown in Figure 1, is obtained using the first two columns of $\mathbf{F}$ and $\mathbf{Y}$ in (3) and (4), which 'explain' parts of inertia $\sigma_{1}^{2}$ and $\sigma_{2}^{2}$, as indicated on the principal axes, expressed as percentages of the total - in this case $0.881(27.7 \%)$ and $0.675(21.3 \%)$ respectively, so that $49.0 \%$ is explained by the twodimensional map. All computations are performed in R ( $\mathrm{R}$ development core team, 2007), using the ca package developed by Nenadić and Greenacre (2007) - the data and R script for all the computations are provided in Ecological Archives.

The results of CA-raw are, as expected, quite different - see Figure 2. First, the analysis has an extra dimension because there is no 'trivial' solution as in CA-relative. Second, because the absolute abundance levels are now included in the description vector of each site, the very high abundance of species $A$.is, isolated in six sites, is a very prominent feature on the first axis, accounting for $76.1 \%$ of the inertia (this species represents $64 \%$ of the biomass in this data set). We can effectively partial out this obvious feature by looking at a map of dimensions 2 and 3 see Figure 3. The configuration is now more interesting to interpret and shows a 'three-pronged' configuration not unlike that of Figure 1 rotated 90 degrees and reflected. Thus, the 'shape' of the abundances is being recovered after the strong 'size' effect has been removed on the first 
dimension. Figure 4 is an animation which shows the rotation about the second axis of the configuration in three-dimensional space.

Another difference between CA-raw and CA-relative is that the row-column relationship no longer has the barycentric interpretation. In the CA-relative asymmetric map of Figure 1, which has 'row principal' scaling, each site is at the weighted average (i.e., barycenter) of the species points, where the weights are the profile elements for that site. Hence sites are all 'interior' to the configuration of species and are attracted to the species for which they have high relative abundance. In CA-raw, however, the raw abundances are determining the relationship, hence the sites are not necessarily interior to the species. Both maps are biplots, however, and projecting the sites onto directions given by the species points would lead to approximate values in the relative or raw abundance matrix, as the case may be. The success of the recovery is measured by the percentage of inertia explained (see Greenacre (1993, 2007: chap. 13) for a discussion of biplots in the CA context). The fact that a much higher percentage of inertia is explained in Figure 2, compared to Figure 1, should be considered in the light of the fact that axis 1 of the CA-raw map of Figure 2 is almost entirely concentrated on the high-abundance species $A$.is, which is being approximated accurately because a whole axis is devoted to its data. A measure of the inertia explained that is concentrated rather on the other 22 species would be to evaluate the inertia contained in the remaining dimensions from 2 onwards (with value 1.516, or 23.9\% of the total) and then calculate how much of that inertia is explained on dimensions 2 and 3: 0.479 $(31.6 \%)$ and $0.306(20.0 \%)$ respectively - i.e., $51.6 \%$. This percentage is similar to the one of $49.0 \%$ obtained in the CA-relative result in Figure 1.

\section{Discussion and conclusions}

The analysis of raw abundance data in the CA framework is a simple variation of the usual algorithm where abundances are proportioned out with respect to row totals (or column 
totals as the case may be). These are all variations on the theme of weighted principal component analysis, with varying choice of weights that are assigned to the points and varying choice of Euclidean metric to measure interpoint distances. We have shown that the visualization of the raw abundances can be achieved very simply using an almost identical algorithm to regular CA: the raw abundances are divided by a constant, the average abundance per site, and equal weights are allocated to the sites. The visualization of the raw abundances makes sense when the sampling is from equal areas or volumes, as is often the case in ecological research. As an alternative to Bray-Curtis dissimilarities being calculated on raw data (or on power-transformed data, as a form of standardization of high and low abundance species) this raw form of CA can be used to visualize these data, with its inherent standardization by the mean in the chi-square distance. The CA framework is weighted Euclidean, which gives many advantages in terms of sum of squares decomposition of variance (i.e., inertia) and the assurance that the points are embeddable in a Euclidean space for dimension reduction.

\section{Acknowledgments}

This research has been supported by the Fundación BBVA, Madrid, Spain, and the author expresses his gratitude to the Foundation's director, Prof. Rafael Pardo. Partial support of Spanish Ministry of Education and Science grant MEC-SEJ2006-14098 is also acknowledged. This report was written while the author was on leave at Stanford University: thanks to Trevor Hastie and colleagues of the Department of Statistics. 


\section{References}

Greenacre, M. J. (1993). Biplots in correspondence analysis. Journal of Applied Statistics, 20, $251-269$.

Greenacre, M. J. (2007). Correspondence Analysis in Practice. Second Edition. London:

Chapman \& Hall / CRC Press. Published in Spanish translation by the Fundación BBVA, Madrid, 2008.

Nenadić, O. and Greenacre, M. J. (2007). Correspondence analysis in R, with two- and threedimensional graphics: The ca package. Journal of Statistical Software, 20 (1).

URL http://www.jstatsoft.org/v20/i03/

Oksanen J., Kindt R., Legendre P. \& O'Hara R.B. (2006). vegan: Community Ecology Package version 1.8-3. URL http://cran.r-project.org/.

R Development Core Team (2007). R: A Language and Environment for Statistical Computing. R Foundation for Statistical Computing, Vienna, Austria.

URL http: / / www.R-project.org 
Table 1: Biomasses of 23 species (columns) observed at 32 sampling sites - only the first five and last five species, and one in the middle (A.is) are shown here.

\begin{tabular}{|c|c|c|c|c|c|c|c|c|c|c|c|c|c|c|}
\hline \multirow[b]{2}{*}{ Sites } & \multirow[b]{2}{*}{ Act } & \multirow[b]{2}{*}{ Pri } & \multirow[b]{2}{*}{ Pol } & \multirow[b]{2}{*}{ Hir } & \multirow[b]{2}{*}{ Pyc } & \multicolumn{2}{|r|}{ SPECIES } & \multirow[b]{2}{*}{... } & \multirow[b]{2}{*}{ C.cr } & \multirow[b]{2}{*}{ Oph } & \multirow[b]{2}{*}{ Ech } & \multirow[b]{2}{*}{ Asc } & \multirow[b]{2}{*}{ Unid } & \multirow[b]{2}{*}{ sum } \\
\hline & & & & & & $\ldots$ & A.is & & & & & & & \\
\hline 3 & 0 & 0 & 3.849 & 0.015 & 0.005 & & 0 & & 0 & 11.187 & 0 & 0 & 0.347 & 24.296 \\
\hline 4 & 0 & 0 & 2.89 & 0 & 0 & & 0 & & 0 & 0.141 & 3.39 & 0 & 0 & 26.316 \\
\hline 5 & 0 & 0 & 2.797 & 0.005 & 0 & & 50.566 & & 0 & 0 & 0 & 0 & 0 & 66.207 \\
\hline 8 & 0 & 0.007 & 2.926 & 0.009 & 0 & & 0 & & 0 & 0.786 & 0 & 0 & 0 & 21.231 \\
\hline 9 & 0 & 0 & 5.268 & 0 & 0 & & 0 & & 0 & 0 & 0 & 0 & 1.586 & 22.645 \\
\hline 10 & 0 & 0 & 6.434 & 0 & 0 & & 0 & & 0 & 0 & 0 & 0 & 0.979 & 43.594 \\
\hline 12 & 0.103 & 0 & 1.992 & 0 & 0.008 & & 127.85 & & 0 & 0 & 0 & 0 & 0 & 152.619 \\
\hline 21 & 0 & 0 & 7.174 & 0 & 0 & & 0 & & 0 & 1.935 & 0 & 0 & 0.231 & 72.505 \\
\hline 25 & 0 & 0 & 6.977 & 0 & 0 & & 0 & & 0 & 0.143 & 0 & 0 & 0 & 32.728 \\
\hline 28 & 0 & 0 & 5.563 & 0 & 0 & & 100.767 & & 0 & 0 & 0 & 0 & 0 & 134.488 \\
\hline 29 & 0 & 0 & 3.542 & 0 & 0 & & 79.17 & & 0 & 0 & 0 & 0.027 & 0 & 111.818 \\
\hline 30 & 0 & 0 & 3.207 & 0 & 0 & & 0 & & 0 & 2.369 & 0 & 0.161 & 0.099 & 36.552 \\
\hline 32 & 0.617 & 0 & 4.956 & 0 & 0 & ... & 0 & ... & 0 & 0.429 & 2.865 & 0 & 0 & 62.147 \\
\hline 33 & 0 & 0 & 4.323 & 0 & 0 & & 0 & & 0 & 0 & 0 & 0 & 0 & 59.911 \\
\hline 34 & 0 & 0 & 6.611 & 0 & 0 & & 380.308 & & 0 & 0 & 0 & 0 & 0 & 420.949 \\
\hline 35 & 0 & 0 & 9.177 & 0 & 0 & & 0 & & 0 & 0 & 0 & 0 & 0.029 & 52.032 \\
\hline 36 & 0.174 & 0 & 2.426 & 0 & 0 & & 0 & & 0 & 0 & 0 & 0 & 0 & 44.426 \\
\hline 49 & 0 & 0.105 & 1.588 & 0 & 0 & & 0 & & 0 & 0 & 0 & 0 & 0.023 & 52.095 \\
\hline 50 & 0 & 0 & 7.007 & 0 & 0 & & 113.701 & & 0 & 0 & 0 & 0 & 0.013 & 170.928 \\
\hline 53 & 0 & 0 & 3.348 & 0 & 0 & & 0 & & 0 & 0 & 0 & 0.034 & 0 & 62.168 \\
\hline 56 & 0 & 0 & 2.454 & 0 & 0 & & 0 & & 11.278 & 0 & 0 & 0 & 0 & 69.777 \\
\hline 57 & 0 & 0 & 7.728 & 0 & 0 & & 0 & & 12.091 & 0 & 0 & 0 & 0.439 & 78.24 \\
\hline 58 & 0 & 0 & 15.791 & 0.063 & 0 & & 0 & & 14.075 & 0 & 0 & 0 & 0.044 & 88.247 \\
\hline 59 & 0 & 0 & 3.655 & 0 & 0 & & 0 & & 0 & 0 & 0 & 0 & 0.776 & 63.451 \\
\hline 73 & 0 & 0 & 1.802 & 0 & 0 & & 0 & & 3.55 & 0 & 2.184 & 0 & 0.137 & 81.604 \\
\hline 74 & 0 & 0 & 1.352 & 0 & 0 & & 0 & & 0 & 0 & 0 & 0 & 0.129 & 75.971 \\
\hline 75 & 0 & 0 & 8.367 & 0 & 0 & & 0 & & 0 & 0 & 0 & 0.087 & 0.413 & 83.904 \\
\hline 76 & 0 & 0 & 3.125 & 0 & 0 & & 0 & & 0 & 0 & 0 & 0 & 0.156 & 80.306 \\
\hline 78 & 0 & 0 & 6.885 & 0 & 0 & & 0 & & 10.741 & 0 & 0 & 0 & 0 & 96.712 \\
\hline 79 & 0 & 0 & 2.73 & 0 & 0 & & 0 & & 0 & 0 & 0 & 0 & 0.183 & 82.491 \\
\hline 83 & 0 & 0 & 2.608 & 0 & 0 & & 0 & & 33.592 & 0 & 0 & 0 & 0.117 & 120.062 \\
\hline 84 & 0 & 0 & 5.052 & 0 & 0 & & 0 & & 7.761 & 0 & 0 & 0 & 0 & 98.768 \\
\hline sum & 0.894 & 0.112 & 153.6 & 0.092 & 0.013 & $\cdots$ & 852.362 & ... & 93.09 & 16.99 & 8.439 & 0.309 & 5.701 & 1331.19 \\
\hline
\end{tabular}


Figure 1: Correspondence analysis of Table 1, with rows in principal coordinates and columns in standard coordinates (row principal asymmetric map). Total inertia $=$ 3.176; inertia explained in the two-dimensional map $=49.0 \%$.

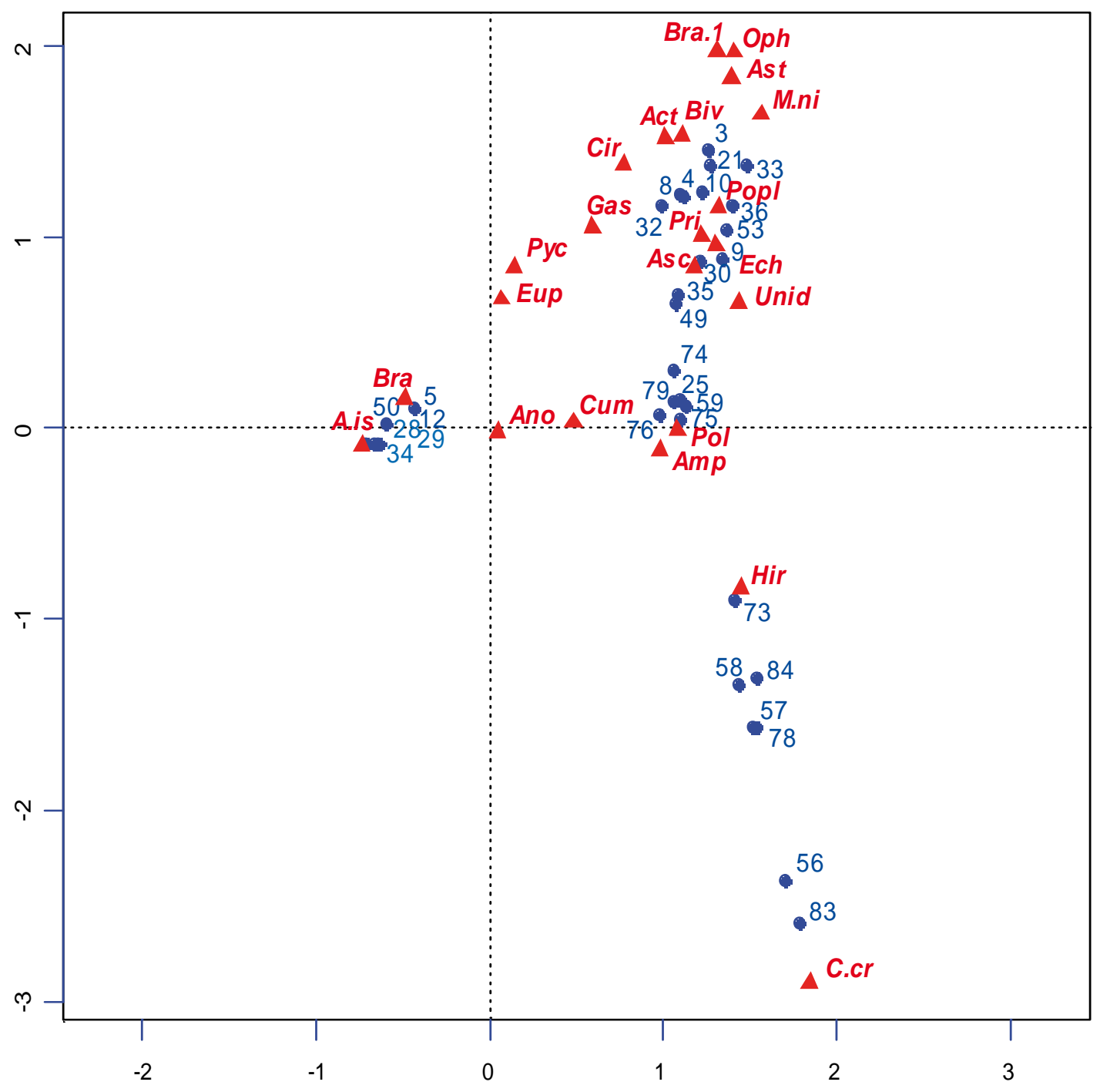


Figure 2: Correspondence analysis of raw abundances in Table 1, with rows in principal coordinates and columns in standard coordinates. Total inertia is 6.341 and inertia explained in the two-dimensional solution is $83.7 \%$.

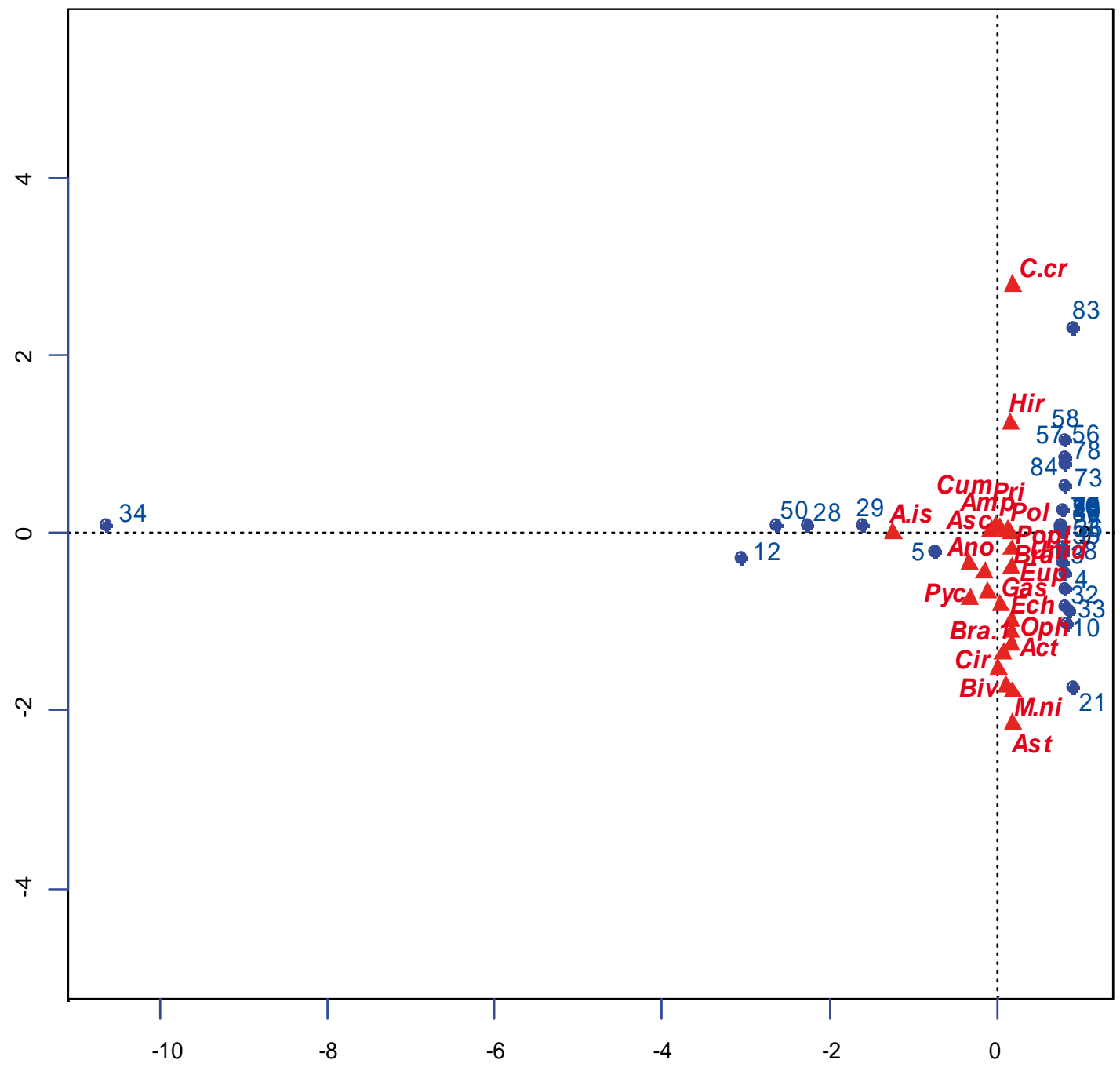


Figure 3: Correspondence analysis of raw abundances in Table 1, showing third dimension along horizontal axis and second dimension vertically.

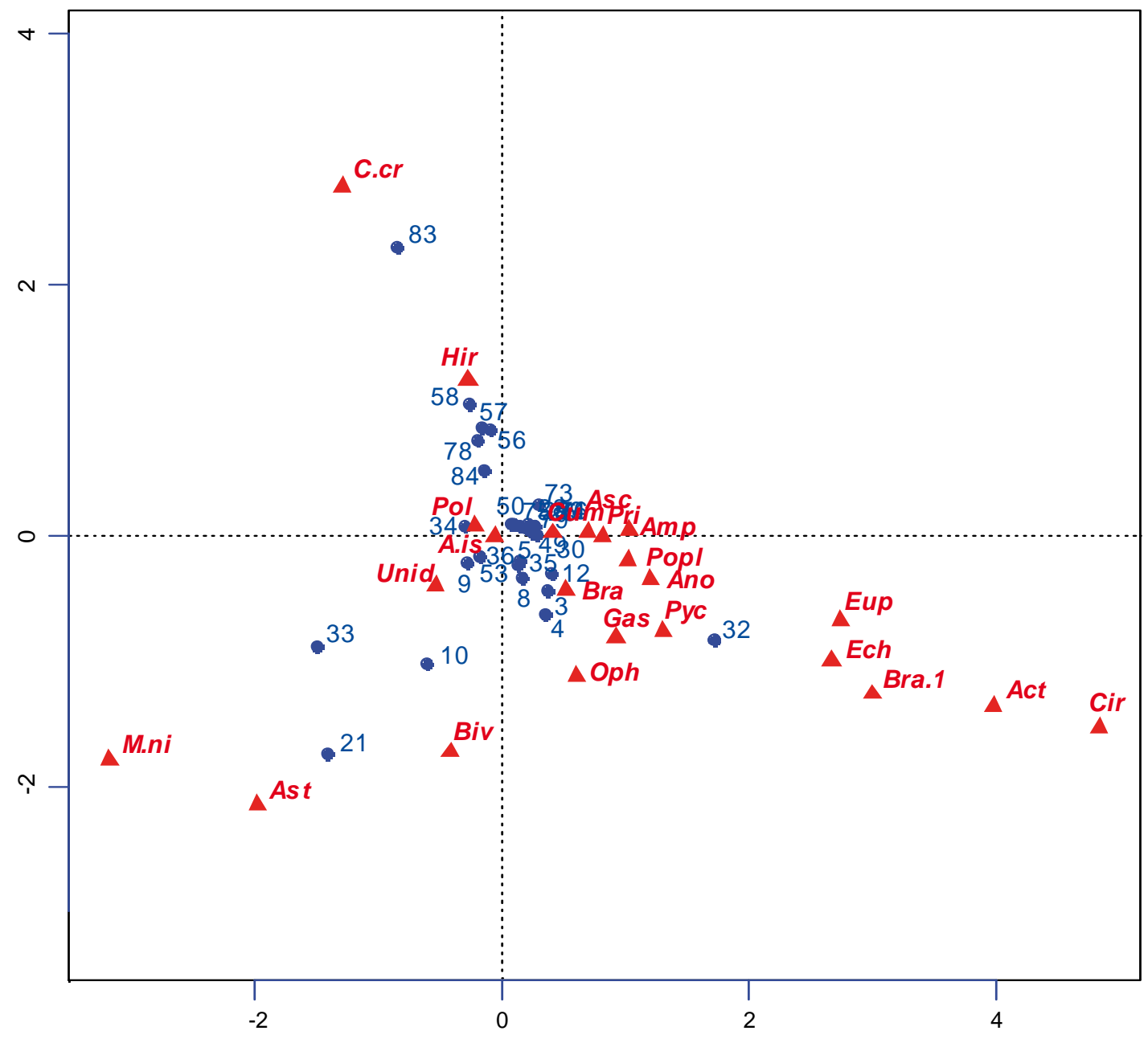


Figure 4: The rotation in three-dimensional space of the CA-raw solution, starting from the principal plane of the first two axes, and then rotating around the vertical second axis to bring in the third axis horizontally. Click on the figure to see the animation.

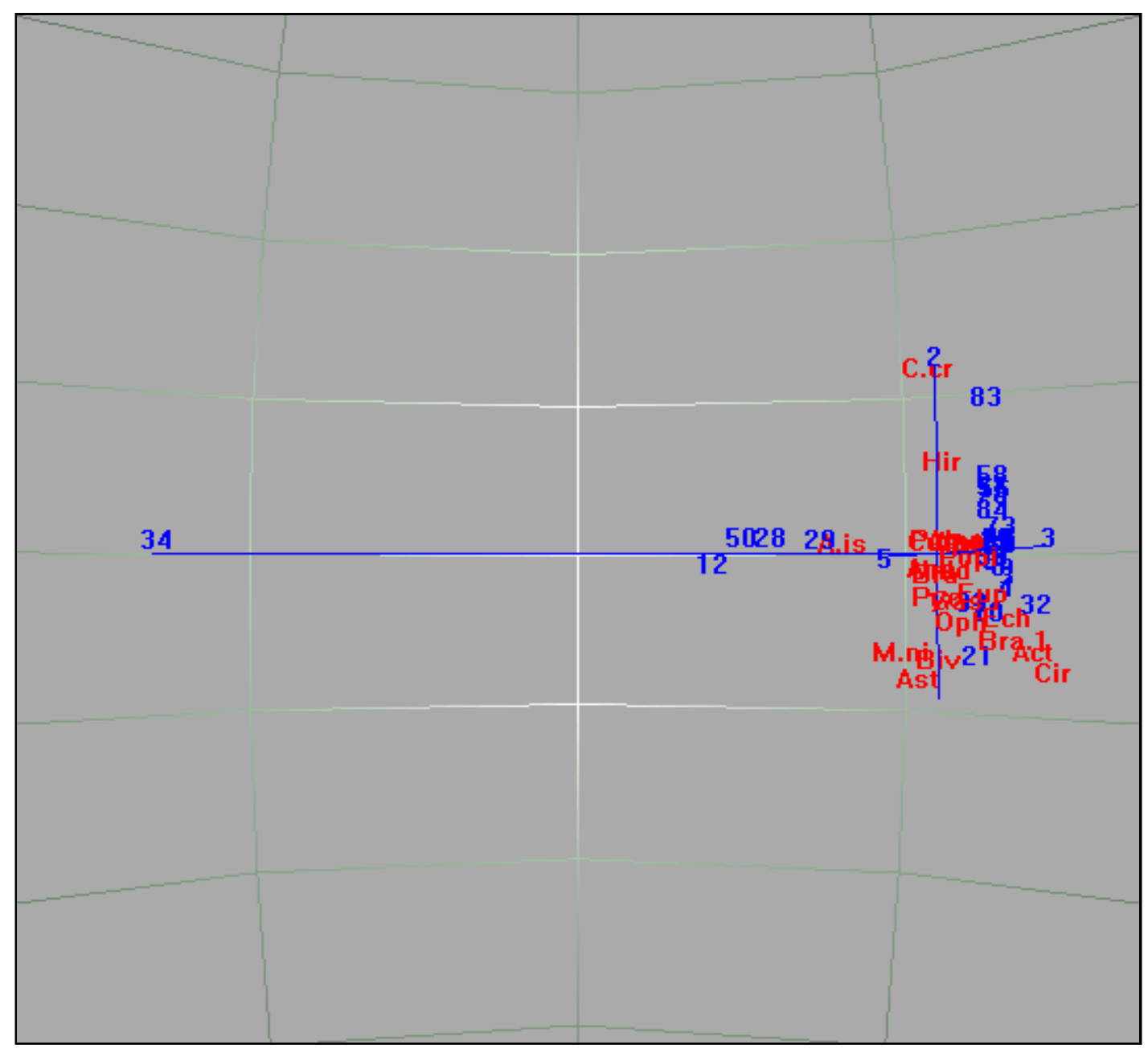

